\title{
Investigação matemática com o GeoGebra em uma propriedade dos polígonos
}

\author{
Duelci Vaz José Eder de Vasconcelos Osni Filho
}

\section{Resumo}

Neste artigo utilizamos a Investigação Matemática com o GeoGebra fundamentada em quatro etapas, a saber: experimentar, conjecturar, formalizar e generalizar para estudar uma propriedade dos polígonos. Identificamos, na condução da atividade, o potencial da proposta, uma vez que nos auxiliou na generalização do resultado a partir de uma álgebra vetorial elementar sobre casos particulares. A utilização do software GeoGebra foi importante na percepção da propriedade, pelas demonstrações visuais que realizamos em diversas situações e conjecturas suscitadas a partir das experimentações.

Palavras-chave: GeoGebra; Investigação Matemática; Propriedade Poligonal.

\section{Abstract}

In this article we perform mathematical research with GeoGebra based on four stages: experiment, conjecture, formalization and generalization to study a property of the polygons. We identify in the conduction of the activity the potential of the proposal, since it helped us in the generalization of a result from an elementary vector algebra on particular cases. The use of the GeoGebra software was important in the perception of the property, by the visual demonstrations that we carried out in diverse situations and conjectures raised from the experiments.

Keywords: GeoGebra; Mathematical research; Polygonal property.

\section{Introdução}

Este trabalho resulta de uma aplicação de uma proposta metodológica para o ensino da Matemática que chamamos de Investigação Matemática com o GeoGebra. Em [4] ilustramos e relatamos uma experiência sobre essa metodologia na exploração de uma propriedade dos determinantes das matrizes quadradas do tipo:

$$
A=\left(a_{i j}\right)_{n \times m}=\left\{\begin{array}{l}
a_{i(j+1)}=a_{i j}+1, \quad \text { para } i=1 \text { e } j=1,2, \ldots n-1 . \\
a_{i j}=a_{i-1} j+n, i=2,3, \ldots n-1, j=1,2, \ldots n-1 .
\end{array}\right.
$$

Concluímos que toda matriz quadrada desta natureza de ordem maior ou igual a 3 possui determinante nulo e no caso especial em que a matriz tem ordem 2, o determinante é sempre -2. Essa 
proposta, que tem uma estreita relação com a Investigação Matemática na Sala de Aula de [1], é novamente utilizada na investigação de uma propriedade dos polígonos, mostrando suas etapas na tentativa de generalizar uma propriedade poligonal. Relatamos nossa experiência na esperança de que esse método possa ser utilizado também por professores de todos os níveis de ensino.

\section{Investigação matemática com o GeoGebra}

Vaz ([4]) estabelece as bases da Investigação Matemática com o GeoGebra que são enunciadas, resumidamente, em quatro etapas: experimentar, conjecturar, formalizar e generalizar o pensamento matemático. Esclarecemos um pouco mais essas etapas aqui, pois elas serão utilizadas no desenrolar desse artigo.

A primeira etapa consiste em explorarmos a capacidade de experimentar que o GeoGebra permite, graças a possibilidade de movimentarmos os entes matemáticos poderemos comparar as representações algébricas e geométricas, percebermos propriedades, compreendermos definições e construirmos conceitos através das percepções obtidas.

A segunda etapa do processo seria levantarmos conjecturas relacionadas à primeira etapa. Conjecturar significa percebermos relações oriundas da experimentação, vislumbrarmos propriedades, relações, resultados gerais importantes para o bom desenvolvimento matemático. Uma vez percebida a conjectura podemos enunciá-la como um resultado a ser investigado, em forma de pergunta ou em forma de problema.

A terceira etapa é a formalização, isto é, a demonstração matemática da conjectura propriamente dita ou a apresentação de uma contra proposição da conjectura levantada, com um argumento pedagógico compatível. Tal atitude é importante, pois não podemos, através da experimentação, aceitar o resultado sob o risco de não estarmos praticando os ideais da Matemática.

A quarta etapa é a generalização. Depois de experimentarmos, conjecturarmos e formalizarmos o saber matemático é importante fazermos a generalização do resultado, quando possível, isto é, investigarmos outras situações pertinentes, situações particulares, enfim, explorarmos o alcance do resultado obtido.

\section{Investigando uma propriedade do triângulo}

Este trabalho começou com um convite para participarmos de um workshop que tinha como tema o uso das tecnologias na Educação Matemática. A ideia era apresentarmos atividades de matemática para um público diversificado usando algum tipo de tecnologia. Utilizar o GeoGebra seria algo interessante para chamarmos a atenção das pessoas que por ali passassem. Assim, procuramos problemas que se encaixassem na proposta da Investigação Matemática com o GeoGebra. Em [2], na seção destinada à soma de vetores, encontramos um problema que despertou nossa atenção: dado um triângulo qualquer, trace os pontos médios de todos os lados e em seguida determine os três vetores com extremidade inicial nos vértices do triângulo e final no ponto médio do lado oposto de cada vértice e demonstre que a soma desses vetores é nula.

O problema mostrou-se apropriado para fazermos uma demonstração visual, ideal para chamar a atenção dos transeuntes no evento. No GeoGebra podemos fazer isso com facilidade, pois o software permite experimentações diversas e com isso fortalecer a ideia de que a proposição é de fato válida ou não. Na tentativa de generalizar a propriedade a utilização da Investigação Matemática com o GeoGebra foi ideal para concluirmos o estudo iniciado com o triângulo.

\section{A resolução do problema e as quatro etapas}

Fase 1: experimentação. Primeiro desenhamos um triângulo qualquer, determinando, em seguida, os pontos médios de cada um de seus lados. Em seguida, traçamos os vetores com extremidade inicial no 


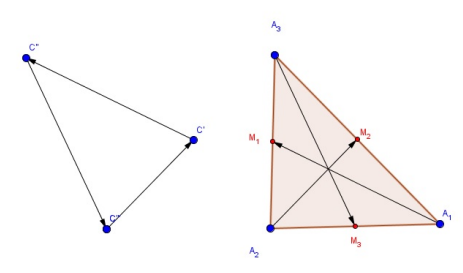

Figura 1: Visualização da construção para triângulos.

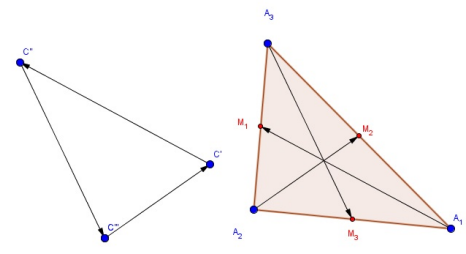

Figura 2: Uma das possibilidades na demonstração visual para o caso do triângulo.

vértice e final no ponto médio do lado oposto, determinando três vetores. Escolhemos um ponto qualquer do plano e transferimos os três vetores, mantendo todas suas características, do seguinte modo: o primeiro vetor, determinado aleatoriamente, deve ser transferido para este ponto; o segundo, também escolhido aleatoriamente, a partir da extremidade do primeiro e o terceiro a partir da extremidade desse último, conforme Figura 1.

Assim, obtemos soma nula, pois os vetores formaram um ciclo fechado. Movimentando qualquer vértice do triângulo verificamos que a configuração geométrica dos vetores se altera, mas a soma continua nula, isto é, o ciclo continua fechado.

Na Figura 2 abaixo, apresentamos outra possibilidade de configuração. No GeoGebra, a experimentação pode ser realizada movimentando os vértices do triângulo e, simultaneamente, a soma dos vetores continua formando ciclo fechado para cada um dos triângulos obtidos. Podemos também habilitar o rastro para vermos o que acontece, o que amplia consideravelmente nossa visão sobre o problema.

Segunda etapa: conjecturar. A partir das experimentações percebemos evidências de que esse é um caso a ser investigado, pois essas demonstrações visuais realizadas sobre vários casos nos permitem pensar que de fato a propriedade é válida para todos os triângulos. Seria interessante fazermos a conjectura em forma de pergunta: será que a soma de todos os vetores com extremidade inicial nos vértices de um triângulo e final no ponto médio do lado oposto é nula? Essa pergunta será respondida de forma investigativa, na etapa seguinte. Terceira etapa: formalização. Agora é necessário fazermos a formalização ou prova analítica do fato. A seguir apresentamos uma possibilidade. Percebemos que a nomeação dos vértices do triângulo da Figura 2, por $A_{1}, A_{2}$ e $A_{3}$ e os pontos médios por $M_{1}, M_{2}$ e $M_{3}$ nos mesmo ajudaria a elaborar um caminho para outros casos, como ficará mais claro posteriormente. Partimos da percepção das seguintes igualdades:

$$
\overrightarrow{A_{1} M_{1}}=\overrightarrow{A_{1} A_{2}}+\left(\frac{1}{2}\right) \overrightarrow{A_{2} A_{3}}, \quad \overrightarrow{A_{2} M_{2}}=\overrightarrow{A_{2} A_{3}}+\left(\frac{1}{2}\right) \overrightarrow{A_{3} A_{1}}, \quad \overrightarrow{A_{3} M_{3}}=\overrightarrow{A_{3} A_{1}}+\left(\frac{1}{2}\right) \overrightarrow{A_{1} A_{2}}
$$




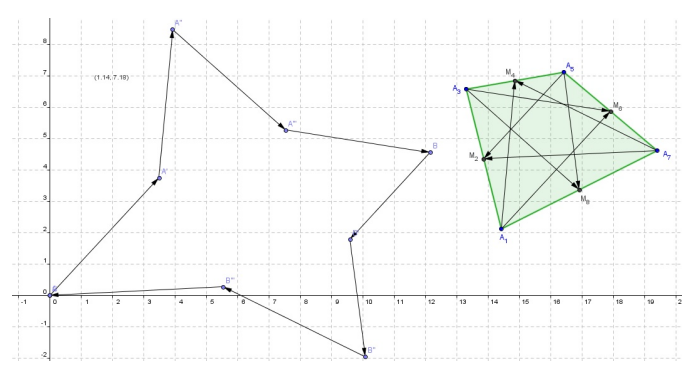

Figura 3: Demonstração visual para o caso de um quadrilátero.

Combinamos membro a membro e obtemos:

$$
\overrightarrow{A_{1} M_{1}}+\overrightarrow{A_{2} M_{2}}+\overrightarrow{A_{3} M_{3}}=\overrightarrow{A_{1} A_{2}}+\overrightarrow{A_{2} A_{3}}+\overrightarrow{A_{3} A_{1}}+\left(\frac{1}{2}\right)\left(\overrightarrow{A_{2} A_{3}}+\overrightarrow{A_{3} A_{1}}+\overrightarrow{A_{1} A_{2}}\right)=\overrightarrow{0}
$$

A soma das parcelas entre parênteses é nula, pois representa um ciclo fechado de vetores, os lados do triângulo.

Quarta etapa: generalização. Daqui para frente desenvolveremos a generalização e para tanto, investigaremos a validade da propriedade para outros polígonos, começaremos pelo quadrilátero convexo e demonstraremos visualmente que a propriedade é válida também para esse caso, como ilustra a Figura 3. Nesta fase, repetimos algumas etapas já mencionadas, como será fácil perceber. Para um quadrilátero não convexo também constatamos visualmente a validade da propriedade, como ilustra a Figura 4.

Diante dessas experiências ficou mais forte a impressão de que a propriedade é válida para todos os quadriláteros. A conjectura para esse caso seria enunciada como segue. Seja $A_{1} A_{2} A_{3} A_{4}$ um quadrilátero qualquer. Tomando-se todos os pontos médios de seus lados e determinando todos os vetores traçados dos vértices aos pontos médios dos lados não adjacentes, a soma desses vetores é nula?

Para demonstrarmos isso, indicaremos por $A_{1}, A_{2}, A_{3}$ e $A_{4}$ os vértices do quadrilátero e por $M_{1}$, $M_{2}, M_{3}$ e $M_{4}$ os pontos médios dos lados $A_{1} A_{3}, A_{3} A_{4}, A_{4} A_{1}$ e $A_{1} A_{2}$, respectivamente, conforme Figura 5 .

A demonstração visual com o GeoGebra revelou que podemos separar os vetores em dois grupos, ambos com soma nula, no caso de um quadrilátero, como mostra a Figura 5. Esse fato foi obtido por experimentação e se constitui fundamental na elaboração de um raciocínio definitivo.

O primeiro grupo é obtido utilizando todos os vetores que têm origem nos vértices do quadrilátero e extremidade final o primeiro ponto médio, não adjacente ao vértice considerado, no sentido horário. Assim:

$$
\begin{aligned}
& \overrightarrow{A_{1} M_{1}}=\overrightarrow{A_{1} A_{2}}+\frac{1}{2} \overrightarrow{A_{2} A_{3}}, \quad \overrightarrow{A_{2} M_{2}}=\overrightarrow{A_{2} A_{3}}+\frac{1}{2} \overrightarrow{A_{3} A_{4}}, \\
& \overrightarrow{A_{3} M_{3}}=\overrightarrow{A_{3} A_{4}}+\frac{1}{2} \overrightarrow{A_{4} A_{1}}, \quad \overrightarrow{A_{4} M_{4}}=\overrightarrow{A_{4} A_{1}}+\frac{1}{2} \overrightarrow{A_{1} A_{2}} .
\end{aligned}
$$

Logo: $\sum_{i=1}^{4} \overrightarrow{A_{i} M_{i}}=\frac{3}{2} \sum_{i=1}^{4} \overrightarrow{A_{i} A_{i+1}}=\overrightarrow{0}$, onde $A_{i+1}=A_{1}$, para $i=4$. O segundo grupo obtido tomando todos os vetores determinados pelos vértices do quadrilátero e o segundo ponto médio 


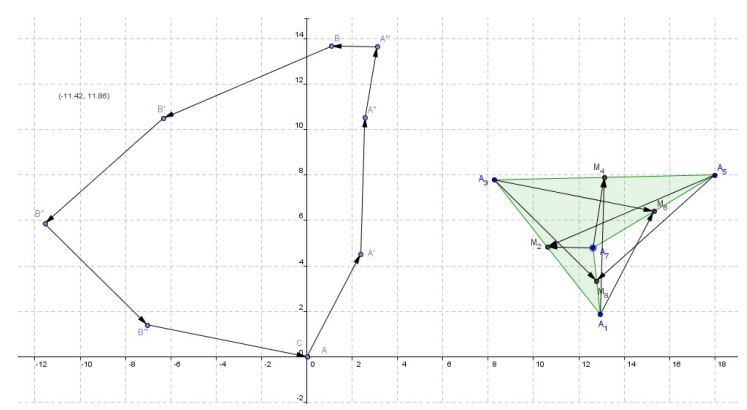

Figura 4: Demonstração visual para o caso de um quadrilátero não convexo.

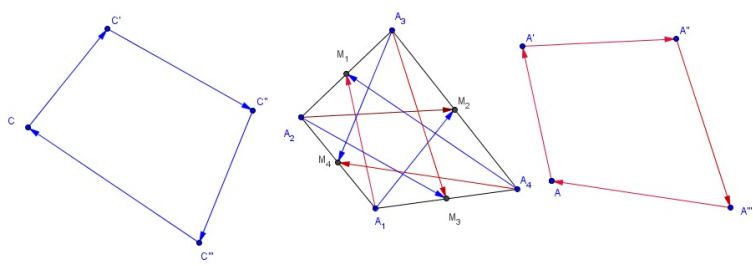

Figura 5: Demonstração visual: vetores separados em dois grupos.

não adjacente, no sentido horário, nos dá:

$$
\begin{aligned}
& \overrightarrow{A_{1} M_{2}}=\overrightarrow{A_{1} A_{2}}+\overrightarrow{A_{2} A_{3}}+\frac{1}{2} \overrightarrow{A_{3} A_{4}}, \quad \overrightarrow{A_{2} M_{3}}=\overrightarrow{A_{2} A_{3}}+\overrightarrow{A_{3} A_{4}}+\frac{1}{2} \overrightarrow{A_{4} A_{1}}, \\
& \overrightarrow{A_{3} M_{4}}=\overrightarrow{A_{3} A_{4}}+\overrightarrow{A_{4} A_{1}}+\frac{1}{2} \overrightarrow{A_{1} A_{2}}, \quad \overrightarrow{A_{4} M_{1}}=\overrightarrow{A_{4} A_{1}}+\overrightarrow{A_{1} A_{2}}+\frac{1}{2} \overrightarrow{A_{2} A_{3}} .
\end{aligned}
$$

Somando os membros das igualdades obtemos para este grupo:

$$
\sum_{i=1}^{4} \overrightarrow{A_{i} M_{i+1}}=\frac{5}{2} \sum_{i=1}^{4} \overrightarrow{A_{i} A_{i+1}}=\overrightarrow{0}
$$

em que: $A_{i+1}=A_{1}$ e $M_{i+1}=M_{1}$, para $i=4$. Assim, obtemos finalmente:

$$
\sum_{i=1}^{4} \overrightarrow{A_{i} M_{i}}+\sum_{i=1}^{4} \overrightarrow{A_{i} M_{i+1}}=\frac{3}{2} \sum_{i=1}^{4} \overrightarrow{A_{i} A_{i+1}}+\frac{5}{2} \sum_{i=1}^{4} \overrightarrow{A_{i} A_{i+1}}=\overrightarrow{0} .
$$

A experimentação nos mostrou que enumerar os vértices e os pontos médios, como ilustrado anteriormente, seria um caminho viável, pois facilitaria a representação simbólica para um polígono com um número arbitrário de vetores. A enumeração também se mostrou eficiente na percepção da composição dos diversos somatórios que iriam aparecer no processo e no encaminhamento de um raciocínio definitivo.

O exemplo a seguir, ilustrado na Figura 6, para um polígono de cinco lados, finaliza a etapa de percepção de propriedades e a partir daí foi possível darmos uma explicação geral para o caso de um polígono de $n$ lados. 


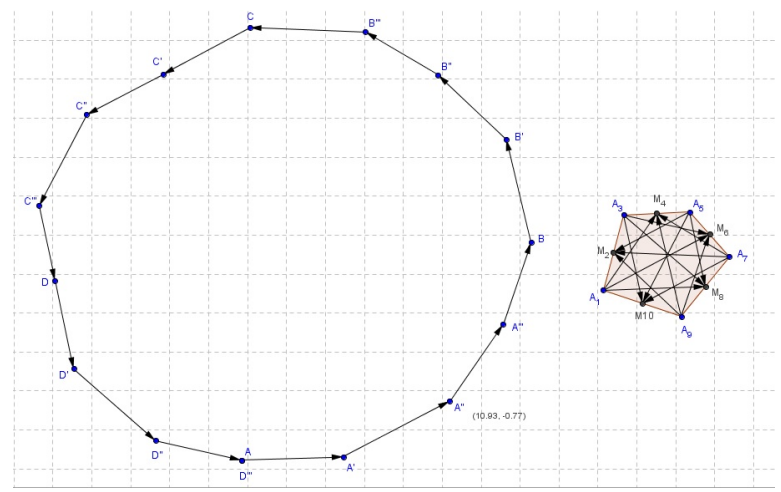

Figura 6: Demonstração visual para o caso de um pentágono.

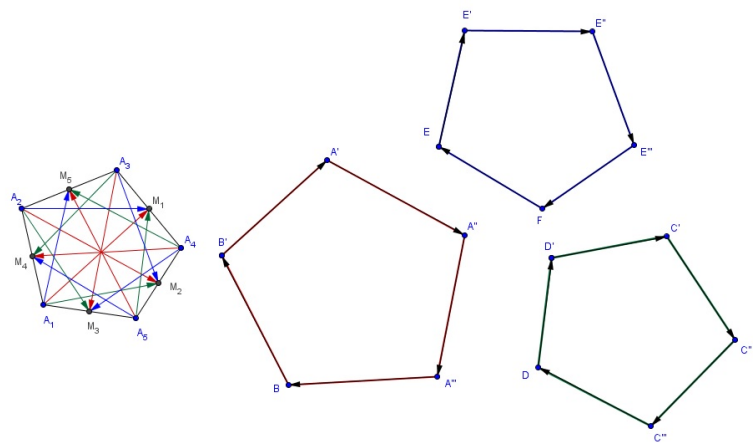

Figura 7: Demonstração visual em três casos para o caso do pentágono.

A estratégia básica mais uma vez foi a de separarmos, neste caso, em três grupos de vetores como ilustrado pela Figura 7, obtida da experimentação com o GeoGebra. Adotamos a estratégia anterior, dividimos este exemplo em três casos. No primeiro grupo, apresentado na Figura 7, obtivemos a seguinte conclusão:

$$
\sum_{i=1}^{5} \overrightarrow{A_{i} M_{i}}=\frac{3}{2} \sum_{i=1}^{5} \overrightarrow{A_{i} A_{i+1}} \text {, em que } A_{i+1}=A_{1} \text { para } i=5 .
$$

Do segundo grupo ilustrado na Figura 7 obtemos:

$$
\sum_{i=1}^{5} \overrightarrow{A_{i} M_{i+1}}=\frac{5}{2} \sum_{i=1}^{5} \overrightarrow{A_{i} A_{i+1}} \text {, em que } A_{i+1}=A_{1} \text { e } M_{i+1}=M_{1} \text { para } i=5 .
$$

E, por fim, o terceiro grupo, também apresentado na Figura 7, nos dá:

$$
\sum_{i=1}^{5} \overrightarrow{A_{i} M_{i+4}}=\frac{7}{2} \sum_{i=1}^{5} \overrightarrow{A_{i} A_{i+1}}
$$

com $M_{i+4}=M_{1}$, para $i=2 ; M_{i+4}=M_{2}$, para $i=3 ; M_{i+4}=M_{3}$, para $i=4 ; M_{i+4}=M_{4} \mathrm{e}$ 

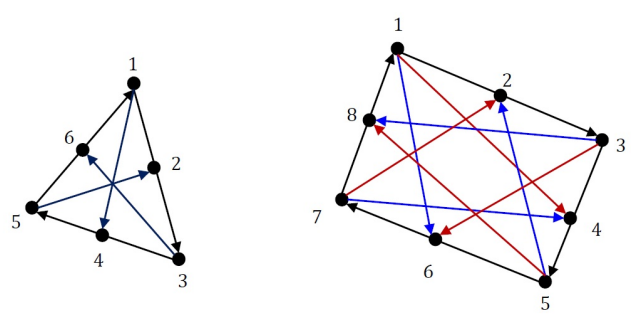

Figura 8: Representação do triângulo e do quadrilátero com a nova notação.

$A_{i+1}=A_{1}$, para $i=5$. Combinando esses resultados obtemos a finalização do argumento, isto é,

$$
\sum_{i=1}^{5} \overrightarrow{A_{i} M_{i}}+\sum_{i=1}^{5} \overrightarrow{A_{i} M_{i+1}}+\sum_{i=1}^{5} \overrightarrow{A_{i} M_{i+2}}=\frac{3}{2} \sum_{i=1}^{5} \overrightarrow{A_{i} A_{i+1}}+\frac{5}{2} \sum_{i=1}^{5} \overrightarrow{A_{i} A_{i+1}}+\frac{7}{2} \sum_{i=1}^{5} \overrightarrow{A_{i} A_{i+1}}=\overrightarrow{0} .
$$

Agora podemos enunciar o fato geral para um polígono de $n$ lados. Considerando os $n-2$ grupos em que os vetores do $k$-ésimo grupo são do tipo: $\overrightarrow{A_{i} M_{i+k}}$, onde $1 \leq k \leq n-2$.

$$
\begin{aligned}
& \sum_{i=1}^{n} \overrightarrow{A_{i} M_{i}}+\sum_{i=1}^{n} \overrightarrow{A_{i} M_{i+1}}+\sum_{i=1}^{n} \overrightarrow{A_{i} M_{i+2}}+\cdots+\sum_{i=1}^{n} \overrightarrow{A_{i} M_{i+n-2}} \\
& =\frac{3}{2} \sum_{i=1}^{5} \overrightarrow{A_{i} A_{i+1}}+\frac{5}{2} \sum_{i=1}^{5} \overrightarrow{A_{i} A_{i+1}}+\cdots+\frac{2 n-3}{2} \sum_{i=1}^{n} \overrightarrow{A_{i} A_{i+n-3}}=\overrightarrow{0},
\end{aligned}
$$

em que $M_{i+1}=M_{1}, M_{i+2}=M_{2}, \ldots, M_{i+n-2}=M_{n-2}$, para $i=n$ e $A_{i+1}=A_{1}, A_{i+2}=A_{2}, \ldots$, $A_{i+n-2}=A_{n-2}$, para $i=n$. Mais geralmente, quando $i+k>n, A_{i+k}=A_{i+k-n}$ e $M_{i+k}=M_{i+k-n}$.

Observamos que para os quadriláteros teríamos oito vetores internos (aqueles que não são lados), para o pentágono teríamos quinze, para um hexágono teríamos vinte e quatro vetores. É fácil deduzir que a fórmula para contagem do número de vetores internos é dada por $f(n)=n^{2}-2 n$, pois de cada vértice saem $n-2$ vetores, num total de $n$ vértices. Assim, o número é dado pela função $f(n)=(n-2) n=n^{2}-2 n$. Também percebemos que para o triângulo existe apenas um grupo de vetores internos com soma nula. Para o quadrilátero existem dois grupos de vetores internos com soma nula. Para um pentágono conseguimos três grupos de vetores. Assim, para um grupo com $n$ vetores teremos $n-2$ grupos de vetores.

\section{Aprimorando a notação}

Para melhor generalizarmos a propriedade dos polígonos utilizaremos as idéias apresentadas em [3]. Vamos renumerar os pontos necessários conforme Figura 8. Os vértices do quadrilátero com os impares: $1,3,5$ e 7 e os pontos médios com os pares: 2, 4, 6 e 8, no sentido horário. Os vetores seguirão o seguinte critério: se um vetor conecta $i$ a $j$ será nomeado de $v_{i, j}$. Por exemplo, existe um vetor entre 1 e 3 , então, esse vetor será nomeado $v_{1,3}$. Assim teremos, $v_{1,3}, v_{3,5}, v_{5,7}$ e $v_{7,1}$ representando os lados do quadrilátero. Além disso, $v_{1,4}$ e $v_{1,6}, v_{3,6}$ e $v_{3,8}, v_{5,2}$ e $v_{5,8}$ e, finalmente $v_{7,4}$ e $v_{7,2}$ representando os vetores saindo do vértice e chegando ao ponto médio do lado não adjacente a esse vértice. 
Ainda levamos em consideração a Figura 8, reescrevemos as fórmulas estabelecidas anteriormente com essa nova notação. Para o triângulo, teremos:

$$
v_{5,2}=v_{5,3}+\frac{1}{2} v_{3,1}, \quad v_{3,6}=v_{3,1}+\frac{1}{2} v_{1,5}, \quad v_{1,4}=v_{1,5}+\frac{1}{2} v_{5,3} .
$$

Somando membro a membro, obtemos:

$$
v_{5,2}+v_{3,6}+v_{1,4}=v_{5,3}+v_{3,1}+v_{1,5}+\frac{1}{2}\left(v_{3,1}+v_{1,5}+v_{5,3}\right)=\frac{3}{2}\left(v_{5,3}+v_{3,1}+v_{1,5}\right)=\overrightarrow{0} .
$$

Ou de forma mais compacta:

$$
\sum_{i=1}^{3} v_{2 i-1,2 i+2}=\sum_{i=1}^{3} v_{2 i-1,2 i+3}+\frac{1}{2} \sum_{i=1}^{3} v_{2 i+3,2 i+1}=\frac{3}{2} \sum_{i=1}^{3} v_{2 i-1,2 i+3}=\overrightarrow{0},
$$

os índices pertencem ao conjunto dos inteiros módulo 6 .

Para os quadriláteros, obtemos:

$$
\sum_{i=1}^{4} v_{2 i-1,2 i+2}=\sum_{i=1}^{4} v_{2 i-1,2 i+1}+\frac{1}{2} \sum_{i=1}^{4} v_{2 i+1,2 i+3}=\frac{3}{2} \sum_{i=1}^{4} v_{2 i-1,2 i+1}=\overrightarrow{0}
$$

e

$$
\sum_{i=1}^{4} v_{2 i-1,2 i+4}=\sum_{i=1}^{4} v_{2 i-1,2 i+1}+\sum_{i=1}^{4} v_{2 i+1,2 i+3}+\frac{1}{2} \sum_{i=1}^{4} v_{2 i+3,2 i+5}=\frac{5}{2} \sum_{i=1}^{4} v_{2 i-1,2 i+1}=\overrightarrow{0},
$$

onde os índices pertencem ao conjunto dos inteiros módulo 8.

Finalmente para um polígono qualquer de $n$ lados, lembramos que teremos $n-2$ grupos de vetores, que são os seguintes:

$$
\begin{aligned}
\sum_{i=1}^{n} v_{2 i-1,2 i+2}= & \frac{3}{2} \sum_{i=1}^{n} v_{2 i-1,2 i+1}=\overrightarrow{0}, \\
\sum_{i=1}^{n} v_{2 i-1,2 i+4}= & \frac{5}{2} \sum_{i=1}^{n} v_{2 i-1,2 i+1}=\overrightarrow{0}, \\
\sum_{i=1}^{n} v_{2 i-1,2 i+6}= & \frac{7}{2} \sum_{i=1}^{n} v_{2 i-1,2 i+1}=\overrightarrow{0}, \\
& \ldots \\
\sum_{i=1}^{n} v_{2 i-1,2 i+2 n-2}= & \frac{2 n-3}{2} \sum_{i=1}^{n} v_{2 i-1,2 i+1}=\overrightarrow{0} .
\end{aligned}
$$

Em que os índices são tomados no conjunto dos inteiros módulo $2 n$.

Evidentemente que a determinação de uma simbologia apropriada facilita as representações e a história da matemática nos mostra o quanto isso é importante para o desenvolvimento das ideias matemáticas. 


\section{Conclusão}

Concluímos nosso artigo enunciando a validade da propriedade. Dado um polígono qualquer, traçando todos os vetores dos vértices até os pontos médios dos lados não adjacentes e calculando a soma de todos eles, obtemos resultante nula. No desenvolvimento do trabalho, percebemos a importância da utilização das novas tecnologias na Educação Matemática. Em diversas oportunidades foram evidenciadas as potencialidades pedagógicas da Investigação Matemática com o GeoGebra e sua importância na construção do conhecimento matemático: na experimentação, permitindo a visualização dos objetos matemáticos em movimento, na elaboração de conjecturas, na releitura de conteúdos, na formalização e generalização dessas conjecturas. Sobretudo, fica evidenciado, o quanto expande nosso olhar sobre os objetos matemáticos. Em certas ocasiões em que se pretendia desenvolver determinados planejamentos e tarefas, observamos outras propriedades, características que não estavam contidas nestes planejamentos. Assim, trabalhar dentro desta proposta pode nos reservar gratas surpresas. Isso é importante, pois estimula novas investigações.

\section{Referências}

[1] Brocardo, J., Oliveira, H., Ponte, J. P. Investigação Matemática em Sala de Aula. Segunda Edição. Autêntica Editora: Belo Horizonte, 2009.

[2] Simons, G. F. Cálculo com Geometria Analítica. MacGraw Hill: São Paulo, 1987.

[3] Vasconcelos, J. E. S. Álgebras Associadas a Grafos Orientados em Níveis e a Propriedade da Koszulidade. Tese (Doutorado em Matemática) - Universidade Federal de Goiás, Goiânia, 2014.

[4] Vaz, D. A. F. Experimentando, Conjecturando, Formalizando e Generalizando: Articulando Investigação Matemática com o GeoGebra. Em: Educativa. Goiânia. Editora da Pontifícia Universidade Católica de Goiás, jan./jun. V.15. n. 1. p. 39-51, 2012.

Duelci Vaz

Instituto Federal de Educação, Ciência e Tecnologia de Goiás (IFG) Pontifícia Universidade Católica de Goiás $<$ duelci.vaz@gmail.com>

José Eder de Vasconcelos Instituto Federal de Educação, Ciência e Tecnologia de Goiás (IFG) <salvadordevasconcelos@yahoo.com.br>

Osni Filho

Instituto Federal de Educação, Ciência e Tecnologia de Goiás (IFG) <osnifilho@live.com> 